\title{
Using Eye Tracking Approach in Analyzing Social Network Site Area of Interest for Consumers' Decision Making in Social Commerce
}

\author{
Suaini Binti Sura ${ }^{1}$, Nona M. Nistah ${ }^{2}$, Sungwon Lee ${ }^{3}$, Daimler Benz Alebaba ${ }^{4}$ \\ Faculty of Computing and Informatics, Universiti Malaysia Sabah, Sabah, Malaysia ${ }^{1,2,4}$ \\ Department of Convergence Software, Kyungmin University, Gyeonggi-do, South Korea ${ }^{3}$
}

\begin{abstract}
The growing popularity of social network site (SNS) in social commerce (s-commerce) has intensified interest in understanding consumers decision making based on the SNS seller generated content (SGC) and user generated content (UGC). This study examines consumers' decision making while doing online shopping by analyzing both SNS's seller-user generated content on SNS utilizing eye tracking approach. Based on eye tracking experimental with 50 participants, gaze map in term of fixation time were collected and analyzed to measure the order of identified Area of interest (AOI) by which consumer viewed and heat map to measure the consumer intensity when looking at the identified AOIs. The results identify that SCG is most important AOI compare to UGC and that product image and description receive the greatest attention from consumers when making decision. Furthermore, seller information serves as a key entry point for SNS-based commerce based on fixation time. The analysis result shows that there is no significant influence of AOIs order based on consumers' viewed on the intensity which consumers look at the AOIs. The comparison between Facebook and Instagram reveals some substantial differences in mean between AOIs based on fixation time and intensity. The findings suggest several AOIs should be addressed and emphasized for sellers and companies who interested in utilizing SNS for their s-commerce strategy.
\end{abstract}

Keywords-Eye tracking; SNS-based commerce; seller generated content; user generated content; social commerce

\section{INTRODUCTION}

The emergence of social media has facilitated the new way of conducting online commerce, whereby rather than doing commerce (sell and buy), it allows users to socialize and build virtual community to generate better information in order to support their decision on commerce activities [1, 2]. This new way of conducting online commerce is known as social commerce (s-commerce). In other words, s-commerce refers to any commerce activities conducted through web 2.0 tools and social media in consumers' online shopping process includes the interaction between business with their customers (B2C), and customer with other customers (C2C) [2]. The concept of interaction and socialization among s-commerce's consumers empowers the e-WOM creates user generated data (UGC), another source of information required both business and consumer in doing their social commerce decision [2, 3, 4].

Social network site (SNS) (i.e. Facebook, and Instagram) is the most popular platform among social media user. Initially

\footnotetext{
This work was supported by Universiti Malaysia Sabah (SLB0160)
}

SNS was created to build virtual network communities so that user can communicate and share experience, idea, information and common interest as in real-life connection [5]. The increase users of SNS, has boosted the use of SNS as scommerce platform. In the context of s-commerce, SNSs a widely support both B2C and C2C commerce model. For B2C, a company creates it's an own SNS account or page to promote and sell their products or services and usually it is linked to company's website. Company has also taken an opportunity to join SNS marketplace and commerce or retail group. As for C2C, SNS marketplace and group (based on common interest) is the best choice to sell and promote the products and services. This kind of SNS is assisting consumer by offering seller or marketer or produce generated content (SGC) such as product or service and seller information, consumer experience and other s-commerce constructs generated from e-WOM based on reviews, ratings, likes, shares, comments and recommendations $[5,6,7]$. The widely use of SNS for online commerce can be seen through the report by [8] claiming that, in case of Malaysia, 26 million Internet users access to SNS and $41 \%$ of Malaysian use SNS as source of new brand discovery and inspiration for their purchases decision. Therefore in this study, we refer SNS based-commerce as the use of SNS platform for online commerce activities in order to support s-commerce activities.

The increase use of SNS as s-commerce platform has motivated researchers to investigate this phenomenon, particularly the SNS consumers' behavior toward s-commerce decision. Understanding customers' behavior is essential for both business and customer. SNS as source for commercial information and social exchange enrich consumer s-commerce decision that lead to higher positive perception of the consumers to the SNS, products and services, resulting increase in volumes of sales, customer loyalty and decreased costs $[6,9]$. In another words, it helps business to plan its scommerce strategy as well as marketing strategy $[1,9]$.

Previous studies indicated the website consumers' behavior is not only affected by the content itself but also the design of website, and position on screen is a critical, particularly in influencing consumer decision-making [7, 9, 10]. In studying of consumers' behavior related to website design and position on screen, eye tracking is one of the methods used by researchers. Eye tracking tool is used to collect the fixation data based on consumers' gaze on certain area known of area of interest (AOI) [11, 12]. Eye-tracking technique is always 
used because researches believe what a person looking at indicates that a person currently is thinking about or attending to and it leads to purchase decision [13, 14]. Thus, investigating purchase decision related to website design gain mass attentions from researchers. For example, Hwang \& Lee [15], Chae \& Lee [16], Huddleston et al.[17], Maslowska et al [18], and Cortins et al. [19] conducted eye tracking study on AOIs of SGC e-commerce website. Huang \& Kuo [20], Wang et al. [21] and Pavani et al [22] conducted eye tracking study on information presentation, navigation and complexity level and task of e-commerce website. Menon et al. [23], Mikalef et al. [24, 25] and Kumar et al. [26] conducted eye tracking study on the s-commerce context. All studies showed the significant finding of eye-tracking data associate with consumers' purchase decision.

In the context of s-commerce website, the design and position highlight two main components: SGC and UGC from e-WOM among consumers, and these explain the main difference between e-commerce and s-commerce. S-commerce focuses on support of social connection during commerce activity, meanwhile e-commerce focuses on product information and features by seller, and personalized shopping experience [6].The emergence of Web 2.0 technology has triggered the evolution of s-commerce platforms and each platform has its own unique function and design to support scommerce. For instant, SNS mainly known for its' social connection, with more unstructured data sources, limited design for information space and features in facilitating scommerce activities, is difference from s-commerce website which is primarily created for the aim business goal, therefore has less unstructured data source and more business features (i.e. shopping cart, ordering) $[3,6]$.

Despite of significant finding of eye-tracking study related to consumers' purchase decision in the context of e-commerce, there are limited study using eye-tracking approach in the context s-commerce particularly the SNS platform. To date, limited studies related to s-commerce can be found especially on SNS platform. In addition, majority of studies gave most focus on UGC components and less on SGC components as well as the combination of the components. Given 1) the increase popularity of eye tracking method in research related to decision making, 2) the limited amount of empirical researches that has employed eye tracking to examine SNS consumer decision making especially examining both SGC and UGC, and 3) the differences between s-commerce website and SNS, have motivated us to conduct this study. Our purpose of conducting this study is to investigate s-commerce consumers' decision making by analyzing SNS (i.e., Facebook and Instagram) AOIs using eye tracking approach, so that we could propose the most important AOI for SNS based-commerce which has ability to affect consumers' decision making. Therefore, we pose these research questions:

- What are the most important AOI for SNS-based commerce based on gaze maps and heat maps?

- Is there any difference on how SNS consumers perceived AOIs based on the gaze maps and heat maps?

- Is there any different of gaze maps and heat maps between Facebook and Instagram?
In this study, we divide the Facebook and Instagram page to targeted AOIs by considering the SNS layout. We used gaze map of fixation time to investigate the order by which SNS consumers viewed the AOIs and heat map to investigate the intensity of SNS consumers look at the each AOI [11, 12].

This rest of the paper is organized as follows: Section 2 discusses background and related literature on s-commerce decision and the use of eye-tracking in analyzing SNS. Section 3 details the research methodological design. Section 4 presents the results. Section 5 discusses the finding and implication of the finding on theory and practice. Last, Section 6 is conclusion emphasizes the purpose of the research and explains the limitation and recommendation of further study.

\section{BACKGROUND AND RELATED LITERATURE}

\section{A. S-commerce Decisiom}

S-commerce refers to commerce activities conducted using the social media platform that support social interaction activities and formation of UGC through consumers participations in the buying and selling products or services in online environment settings [4, 10]. Thus, social platform supports reviews, ratings, online communities and recommendations) about the products/services enhance the scommerce [7]. The increase popularity of social media usage in s-commerce has attracted many researchers to discover how the social platform shaping the consumer behavior due to the fact understanding consumer behavior particularly, consumer decision making in s-commerce is important for planning a new marketing strategy as well as revise the existing strategy, so that it is oriented to particular market audience directly increase the sale [9]. The five stages model of consumer decision making highlights purchase decision is one of the important process and it is commonly accepted and the decision is not only supported by the content offered by the seller but also UGC formation by customers participation [10, 27].

In the s-commerce, seller quality and product quality play important role in determining consumer purchase decision quality, and product type can moderate almost all the association [28]. Social constructs consist of forum and communities, reviews and rating, and recommendations, which are the source of UGC were found out have significant influence on consumers' purchase decision making [28, 29]. In addition, SNS platform influences consumers' decision making in s-commerce [27]. Huang and Benyoucef [10] investigated the effect of social commerce design factors on the consumer decision and claimed that website or webpage design has considerable effect on consumer behavior. Specifically, web design includes the layout and content are significantly influence consumer purchase decision. Nevertheless, for SNS, the essence of the content design is its UCG [23, 26]. These contents are organized in specific area in SNS page and eye tracking approach always used in order to examine the area $[11,12]$ because eye tracking is one of the process tracking method to determine consumer decision making due to the fact eye movement and gaze can reflect human thought in decision making [20,21]. 


\section{B. Eye Tracking and SNS based-commerce AOI}

In recent years, eye tracking approach is widely used in website design studies. Eye tracking used to observe consumer cognitive process to investigate how specific visual features in webpage influence the eye gaze and movement which directly indicate that a person is currently thinking or attending to can lead to a better understand of decision making process $[13,18]$. This suggest eye movement and gaze patterns are consistent with pattern predict by the decision adopted [12, 20]. Researchers have adopted eye-tracking approach to examine website design because of the two advantages. First, compare to other approaches particularly, questionnaire and interview, eye tracking eliminates the subjectivity of self-reporting data. Second, eye tracking capability captures user reaction to webpage and show the page part that captured most user attentions [21].

In process tracking study, eye movements capture the gaze maps and heat maps which are composed of fixation and saccades $[11,12,30]$.Gaze maps capture sequence of fixation in term of fixation i) duration which captures the duration of AOIs looked at, ii) frequency which measures how many time AOIs visited, and iii) time which tells the order by which users viewed the AOIs $[12,30]$. However, heat maps measure the intensity of people look at the objects recognized as fixation intensities telling us the attention key point of website design $[11,31]$. In the context of web design study, webpage grouped in to regions called AOI in various ways depend on website and research purpose. AOIs are categorized based on certain area (e.g., top, middle or bottom) or specific elements (e.g., logo, picture, description) $[11,20]$. Eye-tracking tool is used in collecting fixation data based on the AOIs to measure user interest $[12,13]$. In the s-commerce context, especially SNSbased commerce, AOIs specific elements are divided into two main parts, SGC consist areas which information provided by the seller, and UGC consist areas which information provided and supported by SNS-based commerce consumers [24, 25].

Eye-tracking studies in the e-commerce context focusing on SGC have shown SGC areas received significant higher attention. The higher level attention on SGC areas associate with human brand has significant influence with perceived purchase decision [16]. Based on fixation count, more attention on product image and description indicate consumers more likely to buy [17]. Based on Cortinas [19], heat maps data showed that product area consist of product name, image, price is likely grab more attention from consumers. For s-commerce platform, some eye tracking studies have investigated the consumer purchase decision based on both SGC and UGC AOIs have been conducted and yielded significant finding. The study found out that UGC areas moderate the effect fixation for SCG area based on fixation duration [15]. Nevertheless, fixation duration and heat maps showed that both UGC and SCG especially, image and description captures more consumers' attention. $[18,24]$, and fixation duration confirmed that consumer spend more attention to positive review [25]. However, lack studies have been undertaken through SNSbased commerce. Menon et al [23] and Kumar [26] have conducted eye tracking study by examining targeted area of Facebook but solely focusing on SGC AOIs. The results indicated fixation duration higher on price placement near image [23] and heat maps showed the placement of the image of human face gets more consumers' intensity and attention [26].

From these literatures, we can conclude examining both SGC and UGC AOI using eye-tracking offered researchers a new unique insight to understand consumer's decision making. However, existing studies have not fully explored the impact both SGC and UGC on consumers' decision making in the context SNS-based commerce. Most studies either using eye tracking to analyze SGC or UCG areas. Thus, we took an opportunity to utilize eye tracking approach in examine both SCG and UGC area in the context SNS-based commerce. Our main challenge is to choose the measurement for our eye tracking assessment. Despite fixation duration has been used widely as a measurement, we used fixation time to capture the order by which users viewed the AOIs because we believe the visual hierarchies are important guide for consumer to find the entry point to the SNS page [11, 12]. Furthermore, longer duration fixation doesn't mean always positive attention, it may cause of confusion, for example consumers need time to digest the information that difficult to understand [11,30]. In addition we used heat maps to measure consumer intensity toward AOIs because it is efficiently more effective for web design $[11,31]$.

\section{RESEARCH METHOD}

\section{A. Subject and Design}

50 participants consisted of staffs and students of Universiti Malaysia Sabah Labuan International Campus (UMSLIC) participated in the lab experiment. The participants were divided into two groups: Facebook and Instagram (25 participants for each group). The respondents' range was in age from $24-36$ years old. All of the respondents have experience using SNS for online shopping. The selection of UMSLIC students and staffs as a sample is related to the research material whereby the material for the research is based on the existing Facebook page "Jualan Borong Barang Terpakai UMSKAL 2012” as SNS-based commerce platform. The main purpose of this page is to facilitate selling and buying of second hand product targeting UMSLIC community. For this research, a new Facebook and Instagram page as stimuli were created based on the existing page and calibrated it with eyetracking device. Fixation time and intensity data were collected for further analysis.

\section{B. Material}

The experimental design was based on two simulated SNS: Facebook and Instagram. To retain the nature looking of the existing SNS and the degree of realism, the primary traits and components are kept by retaining the seven AOIs' locations. The AOIs were divided into two main parts. The first part, SGC, consists of Seller profile and product information. Seller profile, which is AOI reflected the seller information, includes the SNS seller's account photo, profile, and name. By clicking the seller's photo and name, the SNS consumers are linked to the seller's SNS page. Product information comprises four AOIs: the product name, image, price, and description. The second part, UGC includes two AOIs, Likes and Comments, which allow the SNS consumers to express their thought and 
feeling about the product or services. The arrangement of the AOIs follows the layout provided by the SNS itself. We included only three products from the same category for each SNS to limit time spent on the assignment and prejudice in product selection. The product was chosen based on the most popular searches in the existing SNS, which are printers and stationeries. As a result, we designated a printer for Facebook and stationery for Instagram.

\section{Procedure and Task}

The study was conducted within two days, 50 UMSLIC staffs and students were participated in the lab experiment. The first day was for Facebook group consisted with 25 participants and the second day was for Instagram participant with 25 participants. Two days were required in conducting this experiment due to the size of computer lab. The experiment was conducted following three phases:

1) Phase 1 - Respondents were needed to complete a basic questionnaire in order to obtain demographic information, online purchasing experience, and familiarity with SNS for online shopping. We give the respondent 5 minutes to complete the task.

2) Phase 2 - Experiment execution: Respondents were briefed on the experiment prior to its execution. Respondents were informed that their browsing activity is recorded. Respondents were given 5 minutes to browse the SNS page without any intervention from the researcher. In this task, respondents were required to browse to search the product that they intended to buy.

3) Phase 3 - Post-test questioning. Following the experiment, a basic interview was conducted to capture the respondent's answer to the product they intended to purchase.

\section{Method and Meassurement}

The experiment allowed the researcher to obtain eye tracking data. The data were based on gaze maps in term of fixation time and heat maps. For the analysis, the gaze maps and heat maps within the selected AOIs were counted in, therefore fixation outside AOIs treated as outliers [19]. The heat maps in term of fixation intensity were represented using the color. The dark color represented the highest level of fixation and light color represented the lowest level of fixation [21]. For analysis purpose, the heat maps color was coded to 5 scales from the scale 1 represented by the blue light color means the lowest level of fixation to scale 5 represented dark red representing the highest level degree of fixation.

Fixation time was used to measure the AOI order based on consumers' viewed. The fixation sequence was ordered from 1 to 7, with 1 being the AOI that the consumers viewed at first and 7 representing the AOIs that the consumers viewed at last. Descriptive statistic was used to analyze the fixation intensity and fixation time. The Independent Sample T-Test was used to compare the differences in mean of the fixation time and fixation intensity between Facebook and Instagram. The SPSS v21 programme was used to analyze the data.

\section{RESULT}

The eye tracking data from 44 participants were used for further data analysis, with 6 participants' eye-tracking data being omitted due to calibration issues. As a result, the total number of participants was 24 for Facebook and 20 for Instagram. The findings were organized in accordance with the research questions.

RQ1: What is the most important AOI for SNS-based commerce based on gaze maps and heat maps?

Table I and Table II present the descriptive statistic mean and standard deviation of heat maps and gaze maps fixation time. Table I, descriptive statistic mean and standard deviation measure the intensity of consumer look at the AOIs meaning that the highest number of mean implies the AOI receives the most intensity from the consumers and the lower number of mean implies the AOI receives the less intensity from the consumers. Therefor heat maps results indicate product image (Mean=4.59, SD =0.726) received most intensity, product description (Mean 3.18, $\mathrm{SD}=1.514$ ) received acceptable intensity, and both likes (Mean $=1.77$, SD $=.985$ ) and comments $($ Mean $=1.77, \mathrm{SD}=.459)$ received less intensity.

As shown in Table II, the descriptive statistic mean and standard deviation represent the fixation time, which measures the order of AOIs viewed by the consumers, beginning with the AOI viewed first and ending with the AOI viewed last. The AOI viewed first by the consumer is indicated by the lowest number of means, while the AOI viewed last by the consumer is shown by the largest number of means. Therefore the results indicate seller profile (Mean $=1.59, \mathrm{SD}=1.317$ ) is the AOI the consumers viewed first and comments (Mean=6.55, $\mathrm{SD}=$ 1.130) is the AOI the consumers viewed last.

RQ2: Is there any difference on how SNS consumers perceived AOIs based on the gaze maps and heat maps?

\section{TABLE I. HEAT MAP (FIXATION INTENSITY) ON AOIS RESULT}

\begin{tabular}{|l|l|l|}
\hline AOI & Mean & Std. Deviation \\
\hline Seller profile & 2.41 & 1.436 \\
Product name & 2.68 & 1.410 \\
Product price & 2.43 & 1.437 \\
Product image & 4.59 & 0.724 \\
Product description & 3.28 & 1.514 \\
Like & 1.77 & 0.985 \\
Comment & 1.77 & 0.459 \\
\hline
\end{tabular}

TABLE II. GAZE MaP (FixAtion Time) ON AOIs Result

\begin{tabular}{|l|l|l|}
\hline AOI & Mean & Std. Deviation \\
\hline Seller profile & 1.59 & 1.317 \\
Product name & 3.30 & 1.193 \\
Product price & 4.05 & 1.099 \\
Product image & 3.16 & 1.817 \\
Product description & 4.91 & 1.309 \\
Like & 4.50 & 1.798 \\
Comment & 6.55 & 1.130 \\
\hline
\end{tabular}


Table III shows the ranking of AOI from most important to least important for both heat maps and gaze maps (fixation time). It implies a significant difference in how SNS consumers perceive the seller profile. Based on heat maps, consumers regard the seller profile as a less important AOI $($ rank $=5)$, yet in the fixation time, the seller profiles (rank =1) is the first AOI viewed by consumers, indicating that it is the most important AOI. The same is true for comments, which both the heat maps and the fixation time suggest as the less important AOIs.

We further investigated the relationship between gaze maps (fixation time) and heat maps, whether the order of viewed AOIs by consumers has an influence on the intensity which they look at the AOIs. Thus, result in Table IV, the regression coefficient results $(\beta=-0.163, t=-1.075, p>0.05)$ show there is no significant influence of the gaze maps (fixation time) (AOI rank - viewed by consumer) on heat maps (the intensity of consumer look at AOIs).

RQ3: Is there any different of gaze maps fixation time and heat maps between Facebook and Instagram?

T-Test for equality of mean is used to compare the differences in gaze maps (fixation time) and heat maps between Facebook and Instagram. Based on heat maps data, Table V shows the differences between Facebook and Instagram. The results show there are significant difference on fixation intensity between Facebook and Instagram for product name $(\mathrm{t}=-4.581, \mathrm{p}<0.001)$, product price $(\mathrm{t}=-9.334, \mathrm{p}<0.001)$ product image $(\mathrm{t}=10.870, \mathrm{p}<0.001)$, product description $(\mathrm{t}=-$ $8.452, \mathrm{p}<0.001)$ and likes $(\mathrm{t}=9.314, \mathrm{p}<0.001)$. But there is no significant difference for seller profile $(\mathrm{t}=-.726, \mathrm{p}>0.05)$ and comments $(\mathrm{t}=-1.792, \mathrm{p}>0.05)$.

Table VI presents how Facebook and Instagram differ in terms of gaze maps (fixation time). The findings demonstrate that there is a substantial variation in the order in which SNS consumers viewed AOI on Facebook and Instagram for product description $(\mathrm{t}=4.926, \mathrm{p}<0.001)$, but there is no statistically significant difference for others AOIs.

TABLE III. AOI Rank Most Crucial To Less Crucial

\begin{tabular}{|l|l|l|}
\hline \multirow{2}{*}{ AOI } & \multicolumn{2}{|l|}{ Rank } \\
\cline { 2 - 3 } & Heat map & Gaze map (Fixation time) \\
\hline Seller profile & 5 & 1 \\
Product name & 3 & 3 \\
Product price & 4 & 4 \\
Product image & 1 & 2 \\
Product description & 2 & 6 \\
Likes & 6 & 5 \\
Comments & 6 & 7 \\
\hline
\end{tabular}

TABLE IV. AOI RANK Most CRUCial to Less CRUCial

\begin{tabular}{|l|l|l|l|}
\hline \multirow{2}{*}{ AOI } & \multicolumn{3}{l|}{ Heat map } \\
\cline { 2 - 4 } & $\boldsymbol{\beta}$ & $\boldsymbol{t}$ & Sig. \\
\hline Gaze map (Fixation time) & -.164 & -1.075 & .289 \\
\hline
\end{tabular}

\section{DISCUSSION}

The findings reveal that SGC areas, particularly product image and description attracted greater attention from SNSbased commerce consumers as compare to UGC area in making purchase decision. This result is in accordance with the previous studies finding, largely have confirmed that SCG in term of product information namely, product name, image, price and description have significant influence on consumer decision making $[16,17,19]$. The most significant aspects of product information are the product image and description, which have the capacity to increase consumers' trust and confident in the product $[7,17]$. The results from gaze maps (fixation time) indicate that SCG of seller information is the first area visited by the consumers. We believe this result is related to SNS (i.e., Facebook and Instagram) page design specifically, the layout, in which seller information, including seller photo, profile, and name, is displayed at the top of the post. According to Huang and Benyoucef [10] website design is one of the consumers purchase decision determinants. However, the heat maps results show that seller information is the least important area among SCG, meaning that consumers place more emphasis on product information during the product search and purchasing process.

Although previous studies [24, 25] have argued SNS consumers give more focus and attention to UGC areas in making purchase decision, but it contradicts with our findings. This phenomenon can be explained based on (1) the role of UGC itself. In this study, SNS UCG comprises of likes and comments, which are considered as supplementary information that the seller does not supply. SNS consumers prioritize product information; but, if they are dissatisfied or uncertain with the information provided by the seller, or if they require extra clarification, they search it form of likes and comments as claimed by Hwang \& Lee [15] and Maslowska et al. [18] UGC supports and moderates the product information for consumer conducting their decision making. (2) Limitation of SNS page design. SNS page design is different from s-commerce webpage. More social constructs for UGC to promote selling and buying activities can be found on s-commerce websites. Scommerce websites typically include rating and product recommendations features and these features are not available on SNS. Although the likes feature is accessible in SNS, it does not imply purchasing when compared to the rating function, which is generated after the purchase done.

TABLE V. T-TeSt EQUALITY OF MEAN RESUlt For HEAT MaP

\begin{tabular}{|l|l|l|l|l|l|}
\hline AOI & $\boldsymbol{t}$ & $\boldsymbol{d} \boldsymbol{f}$ & $\begin{array}{l}\text { Sig. } \\
(\mathbf{2 -} \\
\text { tailed })\end{array}$ & $\begin{array}{l}\text { Mean } \\
\text { difference }\end{array}$ & $\begin{array}{l}\text { Std. Error } \\
\text { difference }\end{array}$ \\
\hline Seller profile & 0.726 & 40.41224 .586 & 0.472 & .292 & 0.402 \\
Product name & 4.581 & 30.662 & 0.000 & 1.367 & 0.273 \\
Product price & 9.334 & 31.66 & 0.000 & 1.750 & 0.187 \\
Product Image & 10.870 & 27.660 & 0.000 & 3.042 & 0.280 \\
Product & 8.452 & 24.266 & 0.000 & 2.000 & 0.237 \\
description & 9.318 & 29.229 & 0.000 & 2.842 & 0.305 \\
Likes & 1.792 & & 0.080 & 0.558 & 0.312 \\
Comments & & & & & \\
\hline
\end{tabular}


TABLE VI. T-TeSt EQUALITY OF MEAN RESUlt FOR GAZE MAP

\begin{tabular}{|l|l|l|l|l|l|}
\hline AOI & $\boldsymbol{t}$ & $\boldsymbol{d} \boldsymbol{f}$ & $\begin{array}{l}\text { Sig. } \\
(\mathbf{2 -} \\
\text { tailed })\end{array}$ & $\begin{array}{l}\text { Mean } \\
\text { difference }\end{array}$ & $\begin{array}{l}\text { Std. Error } \\
\text { difference }\end{array}$ \\
\hline Seller profile & 0.038 & 41.718 & 0.969 & 0.017 & 0.429 \\
Product name & 1.016 & 41.949 & 0.316 & 0.425 & 0.419 \\
Product price & 0.350 & 41.679 & 0.728 & 1.50 & 0.429 \\
Product Image & 0.957 & 36.200 & 0.345 & 2.00 & 0.209 \\
Product description & 4.926 & 41.996 & 0.000 & 1.800 & 0.362 \\
Likes & 0.475 & 41.753 & 0.637 & 1.142 & 0.298 \\
Comments & 0.828 & 41.971 & .0413 & 0.308 & 0.373 \\
\hline
\end{tabular}

We analyzed whether the order of consumers viewed AOIs has significant effect on the intensity of consumer look at the AOIs. Our findings show that there is no significant effect of the order in which consumers viewed AOIs on the intensity with which they looked at the AOIs, suggesting that consumers' attention on SNS AOIs stems from their own context related to purchasing decision. Purchasing decision making is usually more linked to the material's content and the information consolidation of the products or services [19].

The results of the comparison between Facebook and Instagram show that there are some significant difference in mean on the intensity with which consumers look at product information namely, product name, price, image and description, and likes. We argue that the differences in the goals and features served by each SNS, as well as the manner in which sellers utilize the both SNS to provide information, are the main contributors to this findings. Facebook is mainly used to associate with individuals and brands, helping in enhancing brand by leveraging eWom [32]; however Instagram mainly used to share picture, video or other kind of media, and for each media usually followed by story [32]. Additionally, fixation time results which used to measure the order of AOIs indicate the significant different solely for product description. We believe that the differences in the layout of both SNS's webpages contribute to these findings. On Facebook, the product description displays first, followed by the product image, but it is other way round for Instagram.

The results of this study have important implications for both theory and practices. From a theoretical standpoint, most prior research in s-commerce [15, 23, 24, 25, and 26] adopted eye tracking approached to investigate consumers' decision making in utilization of both SGC and UGC. Despite of significant results of those studies, lack of research was undertaken through SNS platform in particularly examining the UCG components. Therefore, this study attempted to fill this gap by extending Mikalef et al [24] with adaption of SGC and UGC through SNS platform. Our findings pinpoint SGC receives more attention from consumers compare to UGC during performing their decision making. Surprisingly, it contradicts the purpose of the SNS which is promoting sociability, indicating while doing online shopping consumers generally focus on their goal which is to purchase. In addition, UCG which is most important to determine consumers' decision making in s-commerce does not in line with our findings. Thus, our study provides the enrichment to existing literature concerning consumers' decision making in scommerce by highlighting both SGC and UGC in a new related setting called SNS-based commerce. From our findings, we argue (1) the difference of s-commerce platform contributes to different features of UGC and indirectly viewed and perceived differently by consumers and (2) UGC plays a role as supportive, additional or alternative information will be referred by consumers if they unsatisfied or uncertain with product information provided by seller. Further study is needed to acquire better understanding of this occurrence.

From a practical viewpoint, the important of our findings generates insights for who are interested to utilize SNS-based commerce as individual sellers or as a company for scommerce strategy. The results reveal product information especially image and description, grab the most of consumers' intensity and attention, indicating product image as key point of the SNS page. It suggests seller and company should focus on how to present their product image and description in order to grab consumers' attention. Company and seller should utilize any method or technique to highlight the product image. Providing additional support to highlight product image include zoom function, close-up or use of human (model) has proven to attract consumers' attention $[16,33]$. The way in which product descriptions are presented should be emphasized so that they are able to attract consumers' attention; failure to do so will result in consumers diverting their attention to gain additional or alternative information from other sources such as product reviews, which is quite risky and may cause consumers to refrain from making purchases. Therefore, seller and company should provide detail information about the product because it has been proven from previous research asserted the good quality of product description means consumers more likely to buy. [17,18, 25] Although UGC (i.e., likes and comments) component is less important, it is not insignificant. Seller and company should continually monitor and response wisely. Furthermore, seller information is discovered as a critical key entry point for both Facebook and Instagram. As a result, clear and exact seller information is required in order to obtain consumer trust and confidence [17].

\section{CONCLUSION}

In summary, we present a study to investigate s-commerce consumers' decision making by analyzing SNS (i.e., Facebook and Instagram) AOIs using eye tracking approach. We used gaze maps (fixation time) to measure the order by which consumers viewed the AOIs and heat maps (fixation intensity) to measure the intensity of people look at the AOIs. In addition, we compared consumer decision-making on Facebook and Instagram based on gaze maps and heat maps data. The findings revealed that both fixation time and intensity suggest that the most important AOI for consumers during their decision making process is product image and the least important is comments. Our investigation showed that the gaze maps (fixation time) (the rank of AOI viewed by the consumer) has no significant effect on heat maps (the intensity of consumer look at AOIs). Furthermore, a comparison of Facebook and Instagram revealed there is significant difference in consumers' intensity on SCG of product information and UGC of like, as well as a significant difference in gaze maps (fixation time) on product description.

The first limitation is the generalizability of the research mainly sample respondents and SNS applications. The sample 
responders consist only UMSLIC students and staffs, age between 22- 34 years old, do not overall picture the SNS user population particularly Malaysia. Therefore the future research, with more samples represents SNS-based commerce consumers population must be adopted to obtain better understanding on subject matters. Because of the popularity of these applications in Malaysia, this study focused mostly on Facebook and Instagram; however, with the advancement of technology and globalization, certain SNS, such as Tiktok and Pinterest, have begun to gain awareness. Each SNS has its own set of primary function criteria. Therefore, in future research, adopting this research to examine the AOIs of the identified SNS to highlight its' function and criteria. Second, current study focuses on gaze maps (fixation time) and heat maps. In the future study, the use of fixation count, frequency and duration will be needed to measure the degree of attention and focus on AOIs. Thus, comparison study can be conducted to provide deep understanding of SNS consumers' attention. Third, for each SNS, we placed three products in the same category, with the goal of making it easier for consumer to search within a limited time frame. However, this is not really represent the real setting of SNS consists more products from variety categories. For the future research, we explicitly recommend different products from different categories should be included to obtain deep understanding on how SNS user make decision based on product and category differentiation. Despite its limitation, we believe this study is still beneficial for company, business organization, and individuals who are interested in utilizing SNS as one of their s-commerce marketing strategies. For other researchers, this study offers fundamental understanding of consumers' attention on SNS page in context s-commerce as a foundation for future research in this field.

\section{ACKNOWLEDGMENT}

We acknowledge financial support for this research from the Universiti Malaysia Sabah: SLB0160.

\section{REFERENCES}

[1] C. Wang and P. Zhang, The evolution of social commerce: the people,management, technology: and information dimensions. Communications of theAssociation for Information Systems, 2012, 31, $1-23$.

[2] X Lin, Li, Y. Li, \& X. Wang, Social commerce research: Definition, research themes and the trends. International Journal of Information Management, 2017, 37(3), 190-201.

[3] Q.Yan, S. Wu, L. Wang, P. Wu, H. Chen and G. Wei, E-WOM from ecommerce websites and social media: Which will consumers adopt?, Electronic Commerce Research and Applications, 2016, 17, 62-57.

[4] Y. Wang and C. Yu, Social interaction-based consumer decision-making model in social commerce: The role of word of mouth and observational learning. International Journal of Information Management, 2017, 37(3), 179-189.

[5] Z. Sheikh, L.Yezheng, T. Islam Z. Hamead \& I.U. Khan, Impact of social commerce constructs and social support on social commerce intentions, Information Technology \& People, 2019, 32(1), 68 - 93.

[6] H. Han, H. Xu, \& H. Chen, Social commerce: A systematic review and data synthesis. Electronic Commerce Research and Applications, 2018, 30, 38-50.

[7] N. Hanjli, Social commerce constructs and consumer's intention to buy, International Journal of Information Management, 2015, 35, 183-191.

[8] Kemp, Digital 2020: Global digital yearbook. Access from wearesocial. com/special-reports/digital-in-2020.,2020.
[9] E. Mazurova, Exploratory analysis of the factors affecting consumer choice in e-commerce: Conjoint analysis, Journal of Information Systems Engineering \& Management, 2017, 2(2), 12.

[10] Z.Huang and M. Benyoucef, The effects of social commerce design on consumer purchase decision-making: An empirical study, Electronic Commerce Research and Applications, 2017, 25, 40-58.

[11] S. Djamasbi, Eye tracking and web experience, AIS Transaction on Human Computer Interaction, 2014, 6(2), 37-54

[12] B. T. Carter \& S. G. Luke. Best practices in eye tracking research. International Journal of Psychophysiology, 2020, 155, 49-62.

[13] S.F. Yang and H.H Lin, Effects of attribute framing varying with the elaboration in online shopping: An eye-tracking approach. In 2014 47th Hawaii International Conference on System Sciences, 2014, 3083-3092.

[14] T. Friedrich, S. Schlauderer, and S. Overhage, The impact of social commerce feature richness on website stickiness through cognitive and affective factors: An experimental study, Electronic Commerce Research and Applications, 2019, 36, 100861.

[15] Y.M. Hwang and K.C. Lee, How does Consumers' Emotion Affect Visual Attention Patterns in Online Shopping Environments? Emphasis on Eye-Tracking Approach, Recent Advance on Finance Science and Management, 2015, 51-55.

[16] S.W. Chae and K.C. Lee, Exploring the effect of the human brand on consumers' decision quality in online shopping: An eye-tracking approach, Online Information Review, 2013. 37(1), 83-100.

[17] P.Huddleston, B.K. Behe, S. Minahan, and R. T. Fernandez, Seeking attention: an eye tracking study of in-store merchandise displays. International Journal of Retail \& Distribution Management, 2015. 43(6), 561-574.

[18] E. Maslowska, C.M. Segijn, K.A.Vakeel and V. Viswanathan, How consumers attend to online reviews: an eye-tracking and network analysis approach, International Journal of Advertising, 2020, 39:2, 282306.

[19] M. Cortinas, R. Cabeza, R. Chocarro, A. Villanueva, Attention to online channels across the path to purchase: An eye-tracking study, Electronic Commerce Research and Applications, 2019, 36, 100864.

[20] Y.F. Huang and F.Y. Kuo, An eye - tracking investigation of internet consumers' decision deliberateness. Internet Research, 2011, 21(5), 541561.

[21] Q. Wang, S. Yang, M. Liu, Z. Cao and Q. Ma, An eye-tracking study of website complexity from cognitive load perspective, Decision Support Systems, 2014, 62, 1-10.

[22] M. L. Pavani, A. B. Prakash, M.S. Koushik, J. Amudha, and C. Jyotsna, Navigation through eye-tracking for human-computer interface, Information and Communication Technology for Intelligent Systems, 2019, pp. 575-586.

[23] R. V. Menon, V. Sigurdsson, N.M. Larsen, A. Fagerstrøm, and G. R. Foxall, Consumer attention to price in social commerce: Eye tracking patterns in retail clothing. Journal of Business Research, 2016, 69(11), 5008-5013.

[24] P. Mikalef, K. Sharma, I.O. Pappas, and M. N. Giannakos, Online reviews or marketer information? An eye-tracking study on social commerce consumers. In Conference on e-Business, e-Services and eSociety, 2017, 388-399.

[25] P. Mikalef, K., Sharma, I.O. Pappas, and M. Giannakos. Seeking information on social commerce: An examination of the impact of userand marketer-generated content through an eye-tracking study. Information Systems Frontiers, 2020, 1-14.

[26] N. Kumar, V. Maheshwari, and J. Kumar, A comparative study of user experience in online social media branding web pages using eye tracker. In 2016 international conference on advances in human machine interaction (HMI) , 2016, 1-6,

[27] H. A. H Hettiarachchi, C. N. Wickramasinghe, and S. Ranathunga, The influence of social commerce on consumer decisions. The International Technology Management Review, 2018, 7(1), 47-58.

[28] A. Chen, Y. Lu, and S. Gupta, Enhancing the decision quality through learning from the social commerce components. Journal of Global Information Management (JGIM), 2017, 25(1), 66-91. 
[29] R. Shekhar and U.P. Jaidev, Antecedents of online purchase intention in the context of social commerce. International Journal of Applied Management Science, 2020, 12(1), 68-95.

[30] P. Bera, P. Soffer \& J. Parsons. Using eye tracking to expose cognitive processes in understanding conceptual models. MIS Quarterly, 2019, 43(4), 1105-1126.

[31] T. Blascheck, K. Kurzhals, M. Raschke, M. Burch, D. Weiskopf \& T. Ertl. Visualization of eye tracking data: A taxonomy and survey. In Computer Graphics Forum , 2017, 36(8),260-284.
[32] D. Belanche, I. Cenjor, and A. Pérez-Rueda, Instagram Stories versus Facebook Wall: an advertising effectiveness analysis. Spanish Journal of Marketing-ESIC. 2019, 23(1), 69 - 93.

[33] R. Boardman and H. McCormick, The impact of product presentation on decision-making and purchasing. Qualitative Market Research: An International Journal. 2019, 22(33), 365-380. 